\title{
A New Action for the Gravitational Interaction
}

\author{
E. Alvarez ${ }^{1}$ \\ Theory Division, CERN \\ 1211 Geneva 23 Switzerland \\ J. Céspedes \\ Dept. d'Estructura i Constituents de la Matèria \\ Universitat de Barcelona \\ Diagonal 647, 08028 Barcelona, Spain \\ E. Verdaguer \\ Grup de Física Teòrica \\ Universitat Autònoma de Barcelona \\ 08193 Bellaterra, Spain
}

\begin{abstract}
A new action for the gravitational interaction is proposed, which is bounded from below in Riemannian spaces and reduces to the standard Einstein-Hilbert action at low energies. The action uses as essential variables the geodesic two-point distance, which gives it a non-local character. It reduces to the Einstein-Hilbert form in the weak coupling limit, plus quartic corrections suppressed by an inverse power of the Planck mass, $m_{p}^{-1}$, which can be put in the (dimensionally continued) Euler characteristic form by field redefinitions.
\end{abstract}

CERN-TH.6349/91

December 1991

\footnotetext{
${ }^{1}$ Permanent address: Departamento de Física Teórica, Universidad Autónoma de Madrid, 28049 Madrid, Spain
} 



\section{Introduction}

The analysis and determination of the quantum effects on the gravitational interaction is one of the most important open problems in theoretical physics (see for example, refs. [1-3] and references therein). There is every reason to believe, however, that whatever those quantum effects may be, the standard picture of space-time as a smooth manifold will not survive when nature is explored at a distance scale of the order of the Planck length, $\ell_{p} \equiv(8 \pi G)^{1 / 2}$.

In the absence of any relevant experimental information, the answer to the question of what is the best mathematical description of spacetime at those distance scales, depends largely on aesthetical prejudices. We have recently put forward our own point of view ${ }^{[4]}$ in which we postulate that the concept of "event" (primarily defined through the set of its coordinates) still makes sense; and that moreover, the set of all events constitute a metric space in the mathematical sense; i.e., there is a function $d_{x y}$ which satisfies the relevant axioms. This metric space is supposed to represent the semiclassical (when all modes with wavelengths $\lambda<\ell_{p}$ have been integrated out) approximation to the fundamental quantum gravity interaction. An arbitrary neighborhood of a given point will not be homeomorphic to an open set of $\mathbf{R}^{n}$, which means that events are not described in this regime by coordinates. It is, however, possible to define at some points a local dimension field, $n(x)$, through an scaling law (see ref. [4] for details). We did not attempt in this reference, though, to find any fundamental guiding principle (analogous to the equivalence principle) in the construction of the basic action for the bilocal field $d_{x y}$.

We would like here to examine this problem again; to be specific, let us ask the following question: Can we find a fundamental action which uses the two- point distance as its basic variable, reduces to the Einstein-Hilbert form at long distances, and presents some improvement over the latter at short distances? We would also like to reformulate the former problems in the most conservative setting in which space-time is assumed to be a smooth manifold, $M$, equipped with a locally inertial reference system ${ }^{2}, \vec{e}_{a} \in T_{x}(M), a=1, \ldots n$ (i.e. such that $\left.\delta^{a b} e_{a}^{\mu} e_{b}^{\nu}=g^{\mu \nu}\right)$. It is clear that if we are able to solve this question in the smooth setting, a simple modification of the same action will also work for general metric spaces. The aim of the present letter is to show that the answer to this question is a qualified yes.

\section{The polytopic action}

We shall begin by associating to each vector of the vierbein $\vec{e}_{a} \in T_{x}(M)$ a point $P_{a} \in M$, by using the exponential map,

$$
\vec{e}_{a} \in T_{x}(M) \longrightarrow P_{a} \equiv \exp \left(\kappa \vec{e}_{a}\right) \in M
$$

This means the following: we consider the unique geodesic going through the given point $x \in M$, with tangent vector $\kappa \vec{e}_{a}$, and define $P_{a}$ as the point on this geodesic at unit proper distance

\footnotetext{
${ }^{2}$ We shall adopt here the Euclidean viewpoint; that is that all quantities are to be computed using Riemannian structures, performing an analytic continuation on the Green functions at the end of the calculations.
} 


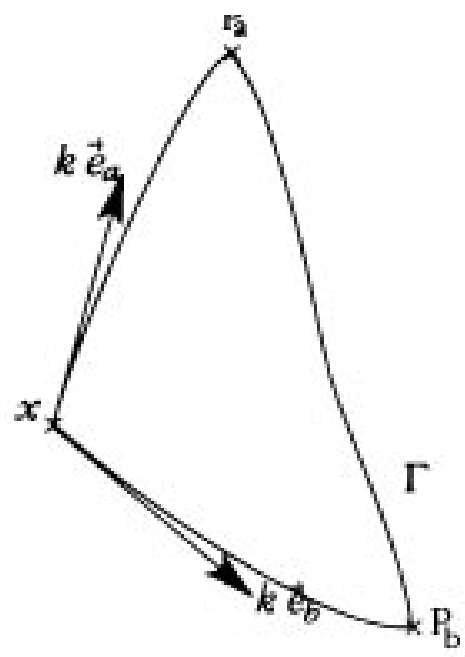

Figure 1

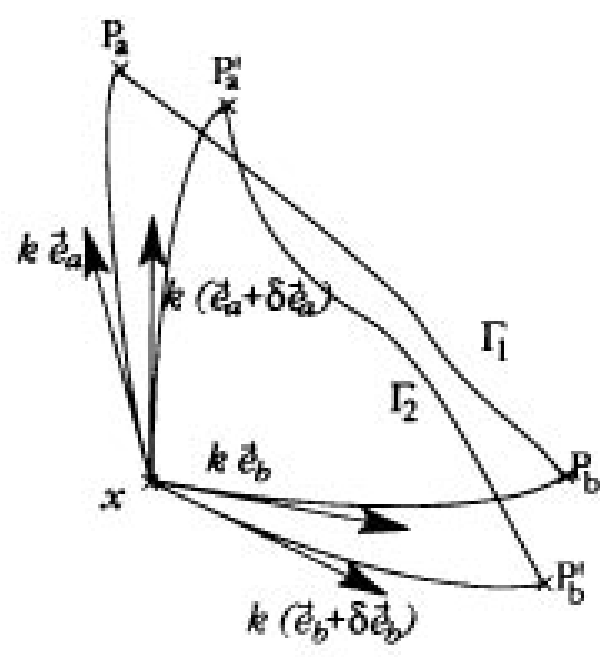

Figure 2

Figure 1: Geodesic $\Gamma$ links points $P_{a}$ and $P_{b}$ at the end of the geodesics with origin at $x$ and tangents $\vec{e}_{a}$ and $\vec{e}_{b}$.

Figure 2: After a variation of the tetrad, the new endpoints are joined by a different geodesic.

from $x$. We have introduced the coupling constant $\kappa$ (with mass dimension -1 ) at this point, in order to get the correct physical dimensions for the coordinates $x^{\alpha}\left(P_{a}\right)$. We have now marked around each point $x \in M$ another $n$ points $P_{a}(x)$ which can be visualized as the vertices of a polytope (that is, a point in dimension $n=1$, a segment in $n=2$, a polygon in $n=3$, a polyhedron in $n=4, \ldots)$.

Let us denote by $\Omega\left(P_{a}, P_{b}\right)$ half the square of the geodesic distance between the points $P_{a}$ and $P_{b}$ :

$$
\Omega\left(P_{a}, P_{b}\right) \equiv \frac{1}{2}\left(\int_{P_{a}}^{P_{b}} d s\right)^{2},
$$

where the line integral has to be evaluated along the (unique) geodesic which passes through both $P_{a}$ and $P_{b}$, as depicted in Fig. 1. This $\Omega$ was called "world function" by Synge ${ }^{[5]}$. The action corresponding to the polytope sitting around the point $x$ is taken to be

$$
\mathcal{S}_{p}[e, x] \equiv \frac{C_{n}}{\kappa^{n+2}} \sum_{a, b} \Omega\left(P_{a}(x), P_{b}(x)\right),
$$

and the total action is just the integral of $\mathcal{S}_{p}(x)$ over the space-time manifold:

$$
S_{p}[e] \equiv \int d^{n} x(e) \mathcal{S}_{p}[e, x],
$$

where $(e)=\operatorname{det}\left(e_{a}^{\mu}\right)$. We have written the constant $C_{n} \kappa^{-n-2}$ in front of the action in order to get $S_{p}$ dimensionless; $C_{n}$ is just a numerical constant.

The action (3) is clearly non local, because $\Omega\left(P_{a}(x), P_{b}(x)\right)$ is defined as an integral involving neighbouring points, and manifestly positive-definite. It remains to be checked whether it 
actually satisfies the conditions we asked for at the beginning of this letter. Before doing that, however, let us point out that our action (3) is not, as such, defined on the orbits of the vierbein field under the action of the orthogonal group $O(n)^{3}$. This means that, in order to get a geometric action (i.e.,, independent of the particular vierbein representative of a given metric), we should average over all elements on each orbit of the relevant group ${ }^{[6]}$.

We shall then take as our fundamental action:

$$
S_{p} \equiv \frac{1}{\operatorname{vol}(O(n))} \int_{O(n)} d \mu(g) S_{p}\left[D_{g} \cdot e\right]
$$

where $d \mu(g)$ represents the Haar measure and $D_{g}$ is the matrix corresponding to $g$ in the fundamental representation of $O(n)$.

\section{Weak coupling}

To explore our action and its relation to the Einstein-Hilbert action, we will expand $S_{p}$ in powers of $\kappa$, assuming that $\kappa$, the coupling constant is small. This will give us the effective action at large distances when compared with $\ell_{p}$, and it is equivalent to a low energy expansion. In the limit $\kappa \rightarrow 0$ our polytope collapses to a point; this means that the weak coupling limit is actually equivalent to an expansion of our action in local terms.

The world function vanishes in the limit $\kappa \rightarrow 0$, called the coincidence limit, but some of its covariant derivatives become local tensors such as the metric and curvature ${ }^{[7,8]}$ :

$$
\begin{gathered}
{[\Omega]=0, \quad\left[\Omega_{; \mu}\right]=0, \quad\left[\Omega_{; \mu \nu}\right]=g_{\mu \nu},} \\
{\left[\Omega_{; \mu \nu \rho}\right]=0, \quad\left[\Omega_{; \mu \nu \rho \sigma}\right]=S_{\mu \nu \rho \sigma} \equiv-\frac{1}{3}\left(R_{\mu \rho \nu \sigma}+R_{\mu \sigma \nu \rho}\right),} \\
{\left[\Omega_{; \mu \nu \rho \sigma \lambda}\right]=\frac{9}{4} S_{\mu \nu(\rho \sigma ; \lambda)},}
\end{gathered}
$$

and the sixth derivative term which can be seen in ref. [7], allowing us to expand $\Omega\left(P_{a}, P_{b}\right)$. The action (3) can be cast in the following form

$$
\begin{gathered}
S_{p}=\frac{1}{\kappa^{n-2}} \int d^{n} x(e)\left[\left(\Lambda-\frac{R}{2}\right)+\kappa^{2} \frac{19 n}{18(n+2)}\left(2 R_{\mu \nu} R^{\mu \nu}+3 R_{\mu \nu \rho \sigma} R^{\mu \nu \rho \sigma}\right)\right. \\
\left.+O\left(\kappa^{4}\right)\right]
\end{gathered}
$$

\footnotetext{
${ }^{3}$ The physical meaning of this is that the action $S_{p}[e]$ breaks Lorentz (rotational) invariance, although this does not happen, as we shall see, in the dominant order in the weak coupling expansion.
} 
where we have defined $\Lambda \equiv \frac{3 n(n-1)}{\kappa^{2}}$, the only contribution of flat space, and neglected a surface term ${ }^{4}$.

Now, the first term, $\kappa^{2-n} \int d^{n} x(e)\left(\Lambda-\frac{R}{2}\right)$, is the Einstein-Hilbert action with cosmological constant if we identify our coupling constant $\kappa$ as $\kappa^{2} \equiv 8 \pi G$, i.e., $\kappa$ is essentially the Planck length. As we claimed, at large distances we recover General Relativity, although a cosmological constant with fixed value appears naturally. Note that this result is obtained in a Riemannian manifold.

The following term, which is the first correction to the Einstein-Hilbert action, is of order $\kappa^{2}$ with respect to the latter; this means that its physical effects are depleted by a factor $\left(\frac{\ell_{p}}{\ell}\right)^{2}$, $\ell$ being the typical length scale of the problem.

It should be kept in mind, however, that when one performs an expansion in terms of a given parameter ( $\kappa$ in our case) there is always an ambiguity, equivalent to a field redefinition of the type

$$
\delta g_{\mu \nu}=a R_{\mu \nu}+b R g_{\mu \nu},
$$

which changes the coefficients of the terms in $R_{\mu \nu} R^{\mu \nu}$ and $R^{2}$ in an arbitrary way ${ }^{[9-11]}$ but does not change the coefficient of $R_{\mu \nu \rho \sigma} R^{\mu \nu \rho \sigma}$. The fact that this coefficient just happens to be non-zero means that the low energy effective action is compatible (to the computed order) with the low energy limit of string theories, and can in particular be put in the (dimensionally continued) Euler characteristic form, by adequately choosing the parameters $a$ and $b$.

\section{The variational principle}

We should now derive the field equations from a variational principle directly from the action (3). The polytopic action, however, being a non- local quantity does not admit the standard variation

\footnotetext{
${ }^{4}$ Were we not willing to average over $O(n)$, we would get an explicit breaking term as a correction of order $\kappa$, namely the first nontrivial correction to the Einstein-Hilbert action would be:

$$
-\frac{\kappa}{4} R_{\mu \nu ; \rho} \sum_{a} e_{a}^{\mu} e_{a}^{\nu} e_{a}^{\rho} .
$$

It is amusing to remark that even in this case, and provided the cosmological constant vanishes, it is easily seen that flat space-time equipped with the rigid cartesian tetrad $\left(e_{a}^{\mu}=\delta_{a}^{\mu}\right)$ is a solution of the theory to all orders in $\kappa$; that is, it is an exact solution, at least in the perturbative sense. Furthermore, as we know explicitly the geodesics of flat space, we can compute the value of $S_{p}[e]$ and find that it is constant regardless of the tetrad chosen (we use Riemannian coordinates around $x$ to represent $P_{a}, P_{b}$ ):
}

$$
\Omega\left(P_{a}(x), P_{b}(x)\right)=\Omega\left(x+\kappa \vec{e}_{a}, x+\kappa \vec{e}_{b}\right)=\Omega\left(x+\kappa L_{c}^{a} \vec{e}_{a}, x+\kappa L_{d}^{b} \vec{e}_{b}\right)
$$

so that our action in flat space is invariant under local changes of the tetrad, $\frac{\delta \Omega}{\delta L}=0$, where $L_{a}^{b} \vec{e}_{b}$ represents any local rotation of the vierbein.

It is also easy to check that if we slightly perturb the tetrad, $e_{a}^{\mu}=\delta_{a}^{\mu}+\kappa \delta e_{a}^{\mu}$, the space geometry changes according to

$$
\delta R_{a}^{\beta}-\frac{1}{2} R_{0} \delta e_{a}^{\alpha}-\frac{1}{2} \delta R \delta e_{a}^{\alpha}=O\left(\kappa^{2}\right)
$$


techniques and we must define what we understand by its variation. From the definition of the world function (1) we have $\Omega\left(P_{a}, P_{b}\right)=\frac{1}{2} \hat{s}_{a b}^{2}$, where $\hat{s}_{a b}$ is the geodesic distance between the points $P_{a}$ and $P_{b}$. The most straightforward definition of $\delta S_{p}$ is,

$$
\delta S_{p}[e]=\frac{3}{\kappa^{2}} \int d^{n} x(e) \sum_{a, b}\left(\hat{s}_{a b}^{2} e_{c}^{\sigma} \delta e_{\sigma}^{c}+2 \hat{s}_{a b} \delta \hat{s}_{a b}\right),
$$

where $\delta \hat{s}_{a b} \equiv \hat{s}_{a b}\left[\Gamma_{2}\right]-\hat{s}_{a b}\left[\Gamma_{1}\right]$. Here $\Gamma_{1}$ is the geodesic through the points prescribed by the tetrads $\kappa \vec{e}_{a}$ and $\kappa \vec{e}_{b}$ by means of the exponential map, and $\Gamma_{2}$ is the perturbed geodesic with end points prescribed by $\kappa\left(\vec{e}_{a}+\delta \vec{e}_{a}\right)$ and $\kappa\left(\vec{e}_{b}+\delta \vec{e}_{b}\right)$; see Fig. 2.

Let an arbitrary geodesic be given by $x^{\mu}=C^{\mu}(s)$, the parameter $s$ will then be fixed, except by the addition of a constant, by

$$
g_{\mu \nu} \frac{d C^{\mu}}{d s} \frac{d C^{\nu}}{d s}=1
$$

From (7) it is clear that the perturbed action can be computed once we know the geodesic to the prescribed points. Now this problem is well posed and has a unique solution if the points are near "enough", or more precisely if a geodesic segment connecting the two points does not cross the cut locus of any of the points ${ }^{[12]}$. What we really need is the geodesic distance, $\hat{s}$, between the two points. We can use the arbitrariness of the parameter $s$ to fix the initial point as $C^{\mu}(0)$, then the end point will be labelled by $C^{\mu}(\hat{s})$. Assuming analyticity and using the geodesic equation recursively we have ${ }^{[13]}$,

$$
\begin{aligned}
& C^{\mu}(0)- C^{\mu}(\hat{s})=\xi^{\mu} \hat{s}-\frac{1}{2} \Gamma_{\alpha_{1} \alpha_{2}}^{\mu} \xi^{\alpha_{1}} \xi^{\alpha_{2}} \hat{s}^{2}+\ldots= \\
&=-\sum_{k=1}^{\infty} \frac{1}{k !} \Gamma_{\alpha_{1}, \ldots, \alpha_{k}}^{\mu} \xi^{\alpha_{1}} \ldots \xi^{\alpha_{k}} \hat{s}^{k},
\end{aligned}
$$

where $\Gamma_{\alpha_{1}, \ldots, \alpha_{k}}^{\mu}(0)$, for $k \geq 2$, are well-known functions involving the Christoffel symbols and their derivatives at the point $C^{\mu}(0), \xi^{\mu}=\left.\left(d C^{\mu} / d s\right)\right|_{s=0}$, and $\Gamma_{\alpha_{1}}^{\mu}=-\delta_{\alpha_{1}}^{\mu}$. The above equation can be solved for $\hat{s}$ and $\xi^{\mu}$ in a recurrent form if we use the formal expansion,

$$
\xi^{\mu}=\sum_{k=0}^{\infty} \xi_{(k)}^{\mu}, \quad \hat{s}=\sum_{k=1}^{\infty} \hat{s}_{(k)} .
$$

It is clear that to first order, if we define $\Delta x^{\mu} \equiv C^{\mu}(0)-C^{\mu}(\hat{s})$, one has,

$$
\xi_{(0)}^{\mu}=\frac{\Delta x^{\mu}}{\Delta x}, \quad \hat{s}_{(1)}=\Delta x
$$

where $\Delta x=\sqrt{g_{\mu \nu} \Delta x^{\mu} \Delta x^{\nu}}$. Substituting (10) into (9) it is not difficult to obtain a recursion formula for $\hat{s}_{(k)}$ and $\xi_{(k-1)}^{\mu}$ given $\hat{s}_{(l)}$ and $\xi_{(l-1)}^{\mu}$ for all $l<k$.

We then have the necessary elements to compute $\delta \hat{s}_{a b}$ in (7) for the geodesic $\Gamma_{1}$, with starting point $C_{1}^{\mu}(0)$, and the perturbed geodesic $\Gamma_{2}$ with starting point $C_{2}^{\mu}(0)$. In practice, however, to perform actual calculations it is simplest to use Riemannian coordinates centered at the point 
$x^{\mu}$. In those coordinates $C_{1}^{\mu}(0)=\kappa e_{a}^{\mu}$ and $C_{1}^{\mu}(\hat{s})=\kappa e_{b}^{\mu}$ and the metric tensor is $\delta_{\mu \nu}$ at $x^{\mu}$. The metric tensor at $C_{1}^{\mu}(0)$ is then ${ }^{[14]}$

$$
g_{\mu \nu}\left(\kappa e_{a}^{\mu}\right)=\delta_{\mu \nu}-\frac{\kappa^{2}}{3} R_{\mu \rho \nu \sigma} e_{a}^{\mu} e_{a}^{\nu}+\ldots
$$

which means that we end up performing a perturbative expansion in $\kappa$. Thus this provides a way of computing the field equations which is alternative to that of the action (6)and at this perturbative level the two procedures are equivalent.

\section{Conclusions}

In conclusion the non-local polytopic action (4) when expanded in powers of its coupling constant leads to (Euclidean) Einstein's equations in the first place with correction terms of the order of the Planck length. Although the expansion would never lead us to believe it, we know that the full action is positive-definite (in Riemannian spaces). We have seen in the last paragraph, however, that it is difficult even to formulate the variational principle in the continuum in a non-perturbative way.

Work is in progress to study a regularized version of our theory in a lattice, in order to check whether the short-distance properties are indeed better than the ones of the standard Einstein-Hilbert theory.

It is already remarkable, however, that an action with the properties we demanded in the introduction exists at all; it can perhaps be taken as an indication that there are still many deep relations to be uncovered in pursuing this line of thought.

\section{Acknowledgements}

We are indebted to L. Alvarez-Gaumé, J.L.F. Barbón, A.H. Chamseddine, P. Hajicek, C.S̃chmid and N. Straumann for many useful suggestions and discussions. This work has been partially supported by CICYT projects.

\section{References}

[1] H.B. Nielsen and M. Ninomiya, Nucl. Phys. B141, 153 (1978).

[2] E. Alvarez, Rev. Mod. Phys. 61, 561 (1989).

[3] E. Alvarez, Phys. Lett. B210, 73 (1988). 
[4] E. Alvarez, J. Céspedes and E. Verdaguer, Phys. Rev. D, in press (1991).

[5] J.L. Synge, Relativity: the General Theory (North Holland, Amsterdam,1960).

[6] J. Preskill, Ann. Phys. 210323 (1991).

[7] J.L. Synge, Proc. Lond. Math. Soc. 32, 241 (1931).

[8] B.S. DeWitt, Dynamical theory of groups and fields, in Relativity, Groups and Topology, ed. by C. DeWitt and B. DeWitt (New York: Gordon and Breach Sc. Pub., 1964).

[9] D.J. Gross and E. Witten, Nucl. Phys. B277, 1 (1986).

[10] B. Zwiebach, Phys. Lett. 156 B, 315 (1985).

[11] S. Deser, Physica Scripta T15, 138 (1987).

[12] S. Kobayashi and K. Nomizu, Foundations of differential geometry (New York: Interscience Pub., John Wiley and Sons, 1963).

[13] L.P. Eisenhart, Riemannian Geometry (Princeton: Princeton University Press, 1950).

[14] A.Z. Petrov, Einstein Spaces (Oxford: Pergamon Press, 1969). 